\title{
The Effectiveness of Physiotherapy in Cervicogenic Headache and concurring Temporomandibular Dysfunction: A Case Report
}

\begin{abstract}
The aim of this case report is to describe the use of physiotherapy interventions in a patient diagnosed with cervicogenic headache $(C G H)$ and temporomandibular dysfunction (TMD). This case study involved a 26 year old male. The subject's cervical and temporomandibular joint (TMJ) active range of movement; passive accessory movements; headache intensity, periodicity and response to analgesics; TMJ clicking and discomfort; Numerical Pain Rating Scale (NPRS) scores, were assessed on initial assessment and on discharge 14 weeks later. The physiotherapy intervention

Lewis F, BSc';
Naude B, MSc ${ }^{1}$
${ }^{1}$ Department of Physiotherapy,
Faculty of Health Sciences,
University of the Witwatersrand.
included cervical and TMJ mobilisation (Maitland approach); trigger point therapy and massage; strengthening of the deep cervical neck flexors and scapulothoracic muscles; and posture correction. The subject improved on all outcome measures and was discharged after nine physiotherapy sessions, with full cervical and TMJ range of movement, improved muscle strength and decreased headache intensity and frequency. This case describes a positive outcome for a patient diagnosed with cervical headaches and temporomandibular dysfunction in which physiotherapy interventions aimed at correcting joint dysfunction, myofascial pain and decreased muscle strength were used effectively.
\end{abstract}

KEYWORDS: CERVICOGENIC HEADACHE, TEMPOROMANDIBULAR DY SFUNCTION, PHY SIOTHERAPY, JOINT MOBILISATION, EXERCISE, CASE STUDY

\section{INTRODUCTION}

Cervicogenic headache $(\mathrm{CGH})$ is headache arising from musculoskeletal disorders of the upper cervical spine and is a common form of chronic recurrent headache (Jull et al 2002). The prevalence of $\mathrm{CGH}$ in the general population has been reported between $0.7 \%$ and $18 \%$ (Bronfort et al, 2004, Jensen 2005, Martelletti and Van Suijlekom 2004). The major criteria for $\mathrm{CGH}$, according to the Cervicogenic Headache International Study Group of the International Headache Society's classification are shown in the Table 1 (Bondi, 2005).

Controversy exists around the cause of $\mathrm{CGH}$, with almost every structure within the cervical spine implicated in some form. Structures identified as

\section{Correspondence to:}

Francoise Lewis

Department of Physiotherapy,

Faculty of Health Sciences,

University of the Witwatersrand

Email: franlewis@live.co.za possible causative agents in $\mathrm{CGH}$, have their sensory innervation through the upper and mid cervical nerve roots (Haldeman and Dagenais 2001). A neuroanatomical and functional relationship exists between the trigeminal and cervical innervated structures accounting for the concurrent craniofascial and cervical pain (Stiesch-Scholz et al 2003). The afferents from C1-3 and the trigeminal nerve afferents converge in the brainstem to form the trigemino-cervical nucleus, which accounts for the referral of pain from the cervical spine structures into the head and face (Bondi, 2005, Haldeman and Dagenais 2001, Jensen 2005, Stiesch-Scholz et al 2003). Any structure innervated by $\mathrm{C} 1-\mathrm{C} 3$ spinal nerves may be the cause of CGH (Jensen 2005). The cervical and paraspinal muscles implicated in CGH, demonstrate findings of muscle dysfunction, trigger points, and decreased cervical muscle strength and endurance (Haldeman and Dagenais 2001, Jull et al 2008). The clinical characteristics of $\mathrm{CGH}$ as described by Davidoff (1998) and
Jensen (2005) are listed in Table 2.

Temporomandibular disorders (TMD) can be defined as a dysfunctional pain phenomenon characterised by temporomandibular joint (TMJ) pain, and cranial-fascial-cervical, preauricular, postauricular, mandibular, temporal, frontal and/or zygomatic arch area pain (Hall 1984), with pain the most frequent motivation for patients with TMD to seek treatment (Türp et al 2007). TMD also referred to as craniomandibular disorders (Costa et al 2008), has a reported prevalence between $3 \%$ and 20\% (Medlicott and Harris 2006, McNeely et al 2005, Svensson 2007).

TMD may be divided into joint related problems caused by internal derangement of the articular disc and/or intraarticular pathology; or muscle or myofascial disorders with trigger points in the muscles of mastication. Patients may present with one type or a combination of both (Davidoff 1998, Türp et al, 2007). Signs and symptoms of TMD are shown in Table 3 (Ciancaglini and Radaelli 2001, Ciancaglini et al 1999, 
Costa et al 2008, Hall L 1984, McNeely et al 2005, Türp et al 2007).

A topographical and functional relationship exists between the cervical spine and the TMJ (Stiesch-Scholz et al 2003). TMD and CGH appear to share many of the same pathophysiological mechanisms and there is overlap between the two conditions (Svensson 2007). Subjects with a TMD often show cervical spine disorders (Stiesch-Scholz et al 2003)

An association exists between headache and TMD but a causal relationship has not been established. Approximately $70 \%$ of subjects complaining of headache also have temporomandibular symptoms (Ciancaglini and Radaelli 2001, Costa et al, 2008). A study by Stiesch-Scholz et al (2003) investigated the prevalence of functional disturbances of the cervical spine in patients with internal derangement of the TMJ in subjects without symptomatic complaints of the cervical spine. The results demonstrated greater restriction of cervical rotation, lateral flexion, and extension, hypomobile functional disturbances of the zygopophyseal joints, and suboccipital, cervical and dorsal shoulder girdle muscle tenderness in patients with internal derangement of the TMJ, when compared to the control group. The neuroanatomical and neurophysiological interconnection between the cervical spine and the orofascial area (De Laat et al, 1998) cause a dysfunctional masticatory system resulting in altered head posture, resulting in pain and dysfunction of the cervical spine (De Laat et al, 1998). There is extensive convergence of afferent input on the trigeminal nuclei which may account for the co-existence of signs and symptoms. Cervical spine sensory information converges with trigeminal afferents within the trigeminal nucleus spinal tract, and fibres arriving in the subnucleus caudalis descend down to $\mathrm{C} 2-\mathrm{C} 3$ and even as far as C6. The $\mathrm{C} 2$ and $\mathrm{C} 3$ ventral cervical nerve roots sensory supply comprises part of the face, including the mandibular angle (De Laat et al 1998).

There are several treatment approaches for the management of CGH (Bronfort et al 2004). Physiotherapy is recommended as a first line of management

Table 1: Cervicogenic Headache International Study Group Diagnostic Guidelines (Bondi, 2005).

Major Criteria of Cervicogenic Headache:

1. Symptoms and signs of neck involvement:

1.1 Precipitation of head pain, similar to the usually occurring one:

- by neck movement and/or sustained awkward head positioning, and/or

- by external pressure over the upper cervical or occipital region on the symptomatic side

1.2 Restriction of range of motion in the neck

1.3 Ipsilateral neck, shoulder, or arm pain of a vague nonradicular nature or,

occasionally, arm pain of a radicular nature

2. Confirmatory evidence by diagnostic anaesthetic blockade

3. Unilaterity of the head pain

\section{Head Pain Characteristics:}

4. - moderate-severe, nonthrobbing, and nonlancinating pain, usually starting in the neck

- episodes of varying duration, or fluctuating, continuous pain

Table 2: Clinical Characteristics of CGH (Davidoff, 1998; Jensen, 2005).

- Frequently thought to be unilateral

- may be on the contralateral side, but there should be one sided dominance

- may occasionally be bilateral, if structures on both sides are affected

- Pain starting in the neck or occipital region, which may spread to occulotemporo-frontal areas

- Pain of deep, nonthrobbing and constant character

- Intermittent attacks lasting hours to days, or constant pain with superimposed attacks of more severe pain

- Headache provoked by neck movements or sustained neck posture

- Ipsilateral nonradicular neck, shoulder or arm pain

- Stiffness and limited cervical range of movement

- Reproduction of headache on palpation of tender areas in the cervical or occipital area

- Autonomic phenomenon including nausea, vomiting, photophobia, dizziness, and ipsilateral blurred vision, and lacrimation (which may be attributed incorrectly to migraines)

(Bondi 2005, Bronfort et al 2004, Jull et al 2002). Studies indicate that musculoskeletal physiotherapy to the cervical spine may prove effective for some headache sufferers in regard to intensity, frequency and duration, if there is an underlying cervical segmental dysfunction present (De Hertogh 2007). Treatment should include joint mobilisa- tion, exercise, stretching and correct ergonomics (Bondi 2005). Multimodal care has both short and long-term maintained benefits for subacute and chronic mechanical neck disorders with or without headaches (Bondi 2005, Gross et al 2004). Management of TMD often involves a multidisciplinary approach, and a conservative approach is the treat- 
Table 3: Temporomandibular signs and symptoms (Costa et al, 2008; Türp et al, 2007; McNeely et al, 2005; Ciancaglini and Radaelli, 2001; Ciancaglini et al, 1999; Hall L, 1984).

- Temporomandibular joint sounds (crepitus or clicking)
- Deeling of stiffness, or fatigue of the jaw
- Difficultly in opening or closing the jaw (trismus)
- Luxation
- Facial Pain, Jaw Pain, Earache
- Headache
- Main on movement of the jaw, joint tenderness
- Vertigo, tinnitus, hypersensitivity to sound, fullness in the ears

Figure 1: Distribution of Symptoms.

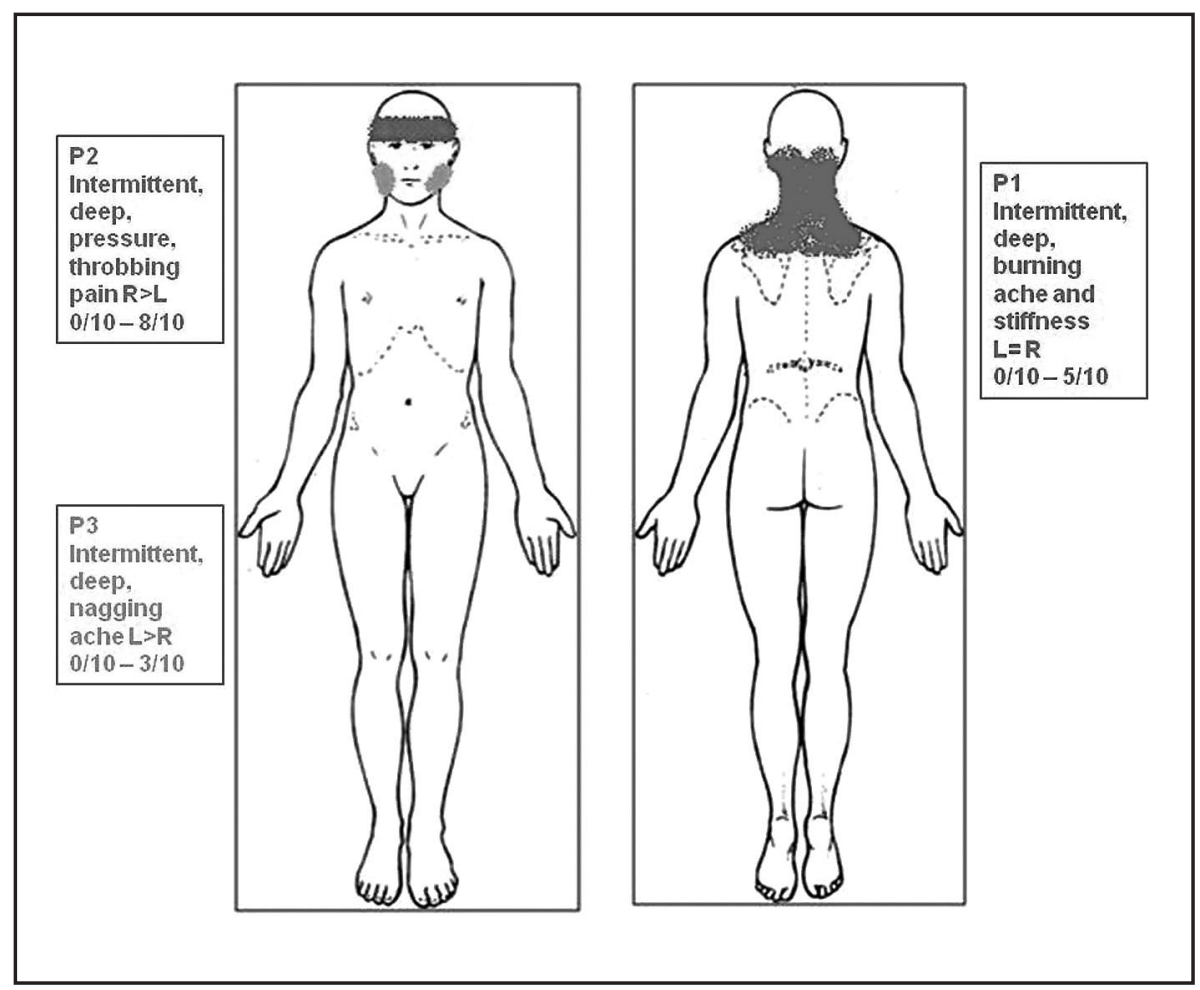

ment of choice (McNeely et al, 2005). The American Academy of Craniomandibular Disorders and the Minnesota Dental Association have recommended physiotherapy as an important treatment modality aimed at restoring oral motor function, relieve musculoskeletal pain and reduce inflammation (McNeely et al, 2005). Physiotherapy programmes are aimed to relax tense muscles, increase ROM, improve muscle coordination and increase muscle strength (McNeely et al, 2005). The aim of this case report is to describe the physio- two years, but worsened since the headaches and cervical pain started. He recalled no previous neck problems but reports previous frequent 'normal headaches'. He was involved in a motor vehicle accident five years ago, in which he sustained a whiplash injury with a complaint of mild cervical stiffness for a couple of weeks. Two years previously he was given a bite plate for the jaw symptoms with no positive effect, so he discontinued its use. The subject recalls no causative incident for the aggravation of symptoms, but reports been under increasing stress at work over the past six months. The cervical pain (P1) and headache (P2) was reported to be aggravated by prolonged postures especially at work, driving and stress; and eased by heat, lying down, stretching, exercise and analgesics. The headache and cervical pain would generally start in the middle of the day and get progressively worse through the day. The subject could not identify definite aggravating factors for the jaw pain (P3), but related it to stress, having a restless night and grinding his teeth. Distribution of symptoms is shown in Figure 1. He reported headaches of severe intensity at least five days a week which when treated with analgesic medication eased but did not clear, with a total pain pattern of 11/15 (Edeling 1998).

\section{Objective Examination}

The subject presented with a forward head posture, bilateral scapula winging and rounded shoulders. At rest he presented with neck pain on shoulder lateral flexion to the right. Other cervical physiological movements revealed minimal limitation. Examination of the TMJ revealed limited opening with deviation to the right, an audible click of the left TMJ, and bilateral TMJ pain. Lateral excursion of the TMJ to the left was limited. TMJ movement did not reproduce headache symptoms.

Passive accessory intervertebral movements (PAIVM's) revealed: O/C1/C2 pain with central and unilateral posteroanterior movements; C1 painful bilaterally with transverse movements to both sides; stiffness with $\mathrm{C} 1 / 2$ movement. O1 posteroanterior movement; $\mathrm{C} 1$ transverse movement bilaterally; and 
Table 4: Treatment intervention with Maitland mobilisation.

\begin{tabular}{|l|l|l|l|l|}
\hline Joint & Mobilisation & Grade & Repetition & Performed at Rx \\
\hline O1 & Postero-anterior & I+ & $10 \times 3$ & $\mathrm{R} \times 1$ \\
& mobilisation & I+ & $30 \times 3$ & $\mathrm{R} \times 2$ \\
& & II+ & $60 \times 2$ & $\mathrm{R} \times 3$ \\
& & IV- & $60 \times 2$ & $\mathrm{R} \times 4-\mathrm{R} \times 7$ \\
& & IV- & $60 \times 4$ & $\mathrm{R} \times 8-\mathrm{R} \times 9$ \\
\hline C1 & Transverse & I+ & $10 \times 3$ & $\mathrm{R} \times 1$ \\
& mobilisation & I+ & $30 \times 3$ & $\mathrm{R} \times 2$ \\
& & II & $60 \times 2$ & $\mathrm{R} \times 3$ \\
& & IV- & $60 \times 2$ & $\mathrm{R} \times 4-\mathrm{R} \times 5$ \\
& & IV- & $60 \times 3$ & $\mathrm{R} \times 6-\mathrm{R} \times 7$ \\
\hline C2 & Central and & II & $30 \times 3$ & $\mathrm{R} \times 2$ \\
& unilateral mobilisation & IV- & $60 \times 2$ & $\mathrm{R} \times 3$ \\
& Unilateral mobilisation & II+ & $60 \times 2$ & $\mathrm{R} \times 4$ \\
& with medial inclination & IV- & $60 \times 3$ & $\mathrm{R} \times 5-\mathrm{R} \times 7$ \\
& & IV- & $60 \times 4$ & $\mathrm{R} \times 8-\mathrm{R} \times 9$ \\
\hline C3 & Central mobilisation & IV- & $60 \times 4$ & $\mathrm{R} \times 8-\mathrm{R} \times 9$ \\
\hline C4-T1 & Central mobilisation & III & $60 \times 3$ & $\mathrm{R} \times 5-\mathrm{R} \times 9$ \\
\hline TMJ & Postero-anterior & $\mathrm{I}+$ & $60 \times 1$ & $\mathrm{R} \times 3$ \\
& Mobilisation of & II & $60 \times 2$ & $\mathrm{R} \times 4$ \\
& right TMJ & IV- & $60 \times 2$ & $\mathrm{R} \times 5$ \\
\hline & Postero-anterior & IV- & $60 \times 3$ & $\mathrm{R} \times 6$ \\
& Mobilisation of & IV- & $60 \times 3$ & $\mathrm{R} \times 6$ \\
& Ieft TMJ & IV- & $60 \times 4$ & $\mathrm{R} \times 7-\mathrm{R} \times 8$ \\
\hline
\end{tabular}

C2 central and unilateral movement reproduced a comparable headache sign. Passive accessory joint movements of the TMJ revealed pain with posteroanterior and lateral movements of the TMJ bilaterally $(\mathrm{L}>\mathrm{R})$. Trigger points and muscle spasm were present in the upper trapezius muscle; levator scapulae muscle; sternocleidomastoid muscle; and the suboccipital muscles. He presented with pain on palpation of the masseter muscle and the medial and lateral pterygoid muscles bilaterally $(\mathrm{L}>\mathrm{R})$. Deep cervical flexor muscle (longus capitis and coli) function was assessed by using the craniocervical flexion test (CCFT) (Jull et al, 2008). He presented with decreased deep cervical flexor muscle recruitment and endurance.

\section{Clinical Reasoning}

The patient met the major diagnostic criteria by the Cervicogenic Headache International Study Group (Bondi 2005), presenting with symptoms and signs of neck involvement, restriction of upper cervical ROM, and bilateral headache with right sided dominance. The CGH was present together with ten- sion-type headaches due to myofascial pain syndrome caused by active trigger points in the muscles supplied by $\mathrm{C} 1$ C3, which contributed to the headaches by referring pain into the head. The structures implicated on examination included the occipital-axial joint; atlanto-axial joint; C2/3 zygopophyseal joint; and the suboccipital, trapezius and cervical paravertebral muscles. These structures all have similar sensory innervation from the $\mathrm{C} 1-3$ cervical nerve roots, which converge with the trigeminal nerve afferents which accounts for the referral of pain from the cervical musculoskeletal structures into the head (Bondi 2005; Jensen 2005; Stiesch-Scholz et al 2003; Haldeman and Dagenais 2001). Physical examination findings confirmed TMD.

\section{Physiotherapy Management}

The patient was diagnosed as having a combined arthrogenous and myofascial TMD and CGH, and an evidence-based treatment was developed.

The cervical joints demonstrating dysfunction $(\mathrm{O} / \mathrm{C} 1 / \mathrm{C} 2 / \mathrm{C} 3$; and C6-T1) and the temporomandibular joint were treated with passive joint mobilisation techniques (Maitland approach). The selection of techniques, grade and rhythm were at the discretion of the treating physiotherapist and based on the physical movement findings (Maitland 1986, McNeely et al 2005, Medlicott and Harris 2006). The mobilisation techniques, grade and repetition performed are presented in Table 4 .

The cervical paraspinal muscles, trapezius and levator scapulae muscles and the muscles of mastication were treated with trigger point therapy and stretching (Davidoff 1998).

Postural correction was addressed (McNeely et al 2005). He was advised regarding his posture at home, at work and in the car. To correct the patient's posture at rest, the scapulothoracic muscles (serratus and trapezius) were strengthened according to the principles documented by Hess (2000).

The deep cervical neck flexors were rehabilitated by the subject performing stabilisation exercises (Jull et al 2008). This initially involved performing a head nod in the supine position. The pressure biofeedback unit was placed in the cervical lordosis and inflated to $20 \mathrm{mmHg}$. The patient watched the dial on the unit to assist in feedback regarding the control of the exercise performed, and was instructed to perform a craniocervical flexion movement. The exercise was progressed to sitting, standing and prone lying. Training of the deep cervical flexor muscles improves deep cervical flexor muscle activity and endurance, and the ability to maintain normal cervical posture in prolonged postural positions (Jull et al 2008). The patient was educated on posture and precipitating factors. He was given a home exercise programme which included posture correction, strengthening exercises to improve the scapula position and deep cervical flexor muscle endurance, as well as stretches for the cervical and upper thoracic muscles.

\section{Outcome}

The patient was treated nine times and was discharged from physiotherapy 14 weeks following the initial assessment. The outcomes of interest included pain, range of motion, oral function and 
patient satisfaction. The outcomes assessed included active cervical and TMJ range of movement; passive accessory intervertebral joint mobilisation of the cervical spine; headache intensity, periodicity and response to analgesia; TMJ clicking and discomfort; subject scores on the NPRS. At the time of discharge the patient's total pain pattern was 4/15 (Edeling 1998); the subject regained full cervical and TMJ range of movement; on palpation had reduced tenderness with PAIVM's of the cervical spine; achieved improved scapula setting and deep cervical flexor muscle endurance; and recorded reduced scores on the numeric pain scale (NPS). TMJ opening was full range with no click audible or pain felt by the patient, mild stiffness of the left TMJ was still occasionally felt.

The overall improvement per treatment was viewed as satisfactory, and the overall outcome was deemed an excellent result. The patient was followed up telephonically three months post discharge and the patient reported that subjectively the improvement gained from physiotherapy intervention had been maintained.

\section{DISCUSSION}

The aim of this case report was to describe the use of physiotherapy intervention in a patient diagnosed with cervicogenic headaches (CGH) and temporomandibular dysfunction (TMD), which is a common presentation in patients seeking treatment. The subject in this case report presented with a two year history of TMD and a more recent six month history of $\mathrm{CGH}$, which was related to increased stress and prolonged postures. Stiesch-Scholz et al (2003) indicate this is a common finding as a functional relationship exists between the cervical spine and TMJ and there is overlap between these two conditions (Svensson 2007). A strong correlation exists between cervical dysfunction and temporomandibular signs and symptoms (Ciancaglini and Radaelli 2001, Vaster et al, 2002).

The headache signs and symptoms were caused by disorders of the musculoskeletal system of the cervical spine, resulting in recurrent headaches. The
TMD presented before the headaches. Whether this is a direct cause effect relationship is difficult to determine. The subject presented with an altered posture which could be attributed to a dysfunctional masticatory system (De Laat et al, 1998). This could make the subject susceptible to the development of cervical spine problems. The co-existence of signs and symptoms could be due to the synergistic or antagonistic relationship between the muscles of the two systems, the altered posture, and the extensive convergence of upper cervical sensory nerve roots and trigeminal afferents in the trigemino-cervical nucleus. Treatment options which have shown a meaningful effect for the treatment of both disorders include: mobilisation, trigger point therapy, low intensity exercise and posture correction (De Hertogh 2007; Bondi 2005; Cameli et al cited by McNeely et al 2005; Jensen 2005; Gross et al 2004; Jull et al 2002; Maitland 1986; Hall 1984).

There were signs of an underlying segmental dysfunction of the upper cervical spine, which should respond well to manual therapy interventions (De Hertogh 2007). Passive joint mobilisation was used to mobilise the hypomobile painful upper cervical zygapophyseal joints (Maitland 1986). Mobilisation has been proposed by Jull et al (2002), De Hertogh et al (2007) and Maitland (1986) to be appropriate intervention for the treatment of $\mathrm{CHG}$. The TMD was treated with passive oscillatory joint mobilisation of the TMJ (Maitland approach) which has been indicated as effective treatment to improve pain and orofascial opening (Cameli et al cited by McNeely et al 2005, Hall 1984). Once the symptoms were settling deep cervical flexor muscle stabilisation exercises as well as scapulothoracic strengthening exercises were introduced. The combined approach of mobilisation and exercise has been shown by Jensen (2005) and Gross et al (2004) to be effective in the management of CGH due to mechanical neck pain. Low intensity endurance exercises to achieve muscle control of the cervicoscapular region have shown to be effective in the treatment of CGH (Jull et al 2002). The active trigger points in the cervical musculature and the muscles of mastication were treated with trigger point therapy which is indicated for the treatment of myofascial pain syndromes by Davidoff (1998). The subject's posture and ergonomics were addressed with education and exercises to correct the alignment of the craniomandibular system and correct the cervical spine alignment as indicated in a study by McNeely et al (2005).

The subject responded well to the treatment for the cervical spine and TMJ indicating effective management with physiotherapy modalities. More than a $50 \%$ improvement in headache frequency was achieved in this subject which is regarded as a clinically relevant result (Jull et al 2002). The TMJ signs and symptoms were reduced with no clicking audible and full TMJ opening without any deviation.

\section{CONCLUSION}

This case report discusses the benefits of a multi-modal physical therapy treatment approach for the treatment of $\mathrm{CGH}$ and TMD which is a common clinical presentation. The combination of joint mobilisation, trigger point therapy; strengthening and postural correction, may be considered appropriate management for this combined condition.

\section{REFERENCES}

Bondi DM 2005 Physical treatments for Headache: A Structured Review. Headache 45:738-746

Bronfort G, Nilsson N, Haas M, Evans RL, Goldsmith CH, Assendelft WJJ, Bouter LM 2004 Non-invasive physical treatments for chronic/recurrent headache. Cochrane Database of Systematic Reviews Issue 3 Art No: CD001878, DOI: 10.1002/14651858 CD001878.pub2

Ciancaglini R, Testa M, Radaelli G 1999 Association of neck pain with symptoms of temporomandibular dysfunction in the general adult population. Scandinavian Journal of Rehabilitation Medicine 31:17-22

Ciancaglini R, Radaelli G 2001 The relationship between headache and symptoms of temporomandibular disorder in the general population. Journal of Dentistry 29:93-98 